\title{
Analytical body forces in numerical actuator disc model of wind turbines
}

Sørensen, Jens Nørkær; Nilsson, Karl; Ivanell, Stefan; Asmuth, Henrik; Mikkelsen, Robert Flemming

Published in:

Renewable Energy

Link to article, DOI:

10.1016/j.renene.2019.09.134

Publication date:

2019

Document Version

Peer reviewed version

Link back to DTU Orbit

Citation (APA):

Sørensen, J. N., Nilsson, K., Ivanell, S., Asmuth, H., \& Mikkelsen, R. F. (2019). Analytical body forces in numerical actuator disc model of wind turbines. Renewable Energy, 147, 2259-2271.

https://doi.org/10.1016/j.renene.2019.09.134

\section{General rights}

Copyright and moral rights for the publications made accessible in the public portal are retained by the authors and/or other copyright owners and it is a condition of accessing publications that users recognise and abide by the legal requirements associated with these rights.

- Users may download and print one copy of any publication from the public portal for the purpose of private study or research.

- You may not further distribute the material or use it for any profit-making activity or commercial gain

- You may freely distribute the URL identifying the publication in the public portal

If you believe that this document breaches copyright please contact us providing details, and we will remove access to the work immediately and investigate your claim. 


\title{
Analytical body forces in numerical actuator disc model of wind turbines
}

\author{
Jens Nørkær Sørensen', Karl Nilsson², Stefan Ivanell², Henrik Asmuth ${ }^{2}$, Robert Mikkelsen' \\ ${ }^{1}$ DTU Wind Energy, Technical University of Denmark, Lyngby, Denmark \\ ${ }^{2}$ Uppsala University, Campus Gotland, Visby, Sweden
}

\section{Corresponding author: Jens N. Sørensen (jnso@dtu.dk)}

\begin{abstract}
An analytical model for representing body forces in numerical actuator disc models of wind turbines is developed and validated. The model is based on the assumption that the rotor disc is subject to a constant circulation modified for tip and root effects. The model comprises expressions for both the axial and the azimuthal force distributions, and is generalized to be utilized for all kinds of inflow, including wind shear, turbulence, and shadow effects in wind farms. The advantage of the model is that it does not depend on any detailed knowledge concerning the wind turbine being analysed, but only requires knowledge regarding the rated wind speed and nameplate capacity. To validate the analytical model, results are compared to numerically generated results using detailed information regarding geometry and airfoil data for the $2 \mathrm{MW}$ Tjaereborg wind turbine and the 10MW DTU reference turbine. The comparisons show very good agreement between the loadings using the new analytical model and the airfoil data based method for the two tested wind turbines, demonstrating that the analytical model represents a simple and reliable way of introducing body forces in actuator disc simulations without any prior knowledge of the wind turbine being analysed.
\end{abstract}

Keywords: Actuator disc, rotor aerodynamics, wind energy, body forces

\section{Introduction}

In rotary aerodynamics, the actuator disc has for many years been employed as a means to include body forces in the energy and momentum equations. It was originaly utilized to include rotor forces in onedimensional momentum and energy analyses of propellers by Rankine [1] and Froude [2], and for establishing conditions for optimum rotors by Betz [3]. The actuator disc has a long history in the aerodynamics of propellers and turbines (see e.g. Horlock [4]) and forms an important ingredient in the bladeelement/momentum approach (Glauert [5]; Sørensen [6]). Today, the actuator disc methodology is often employed in connection with the Navier-Stokes equations as a simple and efficient way of introducing the rotor loading into the flow equations. In numerical computations of e.g. wind farms, it is often impossible to resolve the entire rotor blade geometry of the individual wind turbine, and instead the rotor loading is replaced by appropriately determined body forces. In its simplest version, the actuator disc consists of a constant loading determined from the average thrust acting on the rotor. Comparisons with experiments have demonstrated that the method works well for axisymmetric flow conditions and is capable of providing useful information regarding the overall flow behaviour. However, important flow dynamics is missing if no information regarding the actual loading is included in the forcing. A way to include the actual forcing is to employ a bladeelement approach combined with tabulated airfoil data. The first computations of actual wind turbines employing numerical actuator disc models in combination with a blade-element approach were done by Sørensen et al. [7]-[8], and later by Masson et al. [9] to study unsteady flow phenomena. Masson and colleagues 
[10] also devised techniques for employing the actuator disc model to study wake interactions in wind farms. The first comprehensive and systematic large eddy simulations of wind farms in the atmospheric boundary layer using an actuator disc approach forwind turbines are due to Jimenez et al. [11], Calaf et al. [12] and PortéAgel et al. [13]. Today, the actuator disc technique has been supplemented by more sophisticated methods, such as the actuator line method (Sørensen and Shen [14]) and the actuator surface method (Shen et al. [15]). In spite of their approximate nature, the actuator methods in general exhibit a good numerical accuracy and they are much faster than conventional mesh-based rotor computations.

The most simple way of implementing body forces in the Navier-Stokes equations usig the actuator disc concept is to let them be prescribed either as constant loadings or as having a prescribed radial distribution. For an actual wind turbine, however, this may not comply with the actual shape of the load distribution. In a recent study by Simisiroglou et al. [16], where different prescribed axial loadings were tried, it was found that differences in the shape of the force distribution could be felt in the wake at least five diameters downstream of the turbine. This will have an impact on the inflow conditions to the surrounding turbines in a wind farm. Moreover, as was demonstrated by Porté-Agel et al. [13], it may also be important to include the azimuthal force distribution in the simulations to capture correctly the near-wake behaviour. However, to do this adequately would require full knowledge of the actual geometry, access to airfoil data, and information regarding the operational envelope of of the wind turbine. A systematic study on different ways to include body foces was recently carried out by van der Laan et al. [17], in which it was shown that knowing the details of the actual loading results in a much more reliable computation of the wake than simply assuming some more or less arbitrary shapes. A problem, however, is that it in most cases only crude information is known about the actual turbine, either because geometry and airfoil data are confidential or simply because the developer has not yet decided size and type of the turbines in the initial development phase of a wind farm. There is therefore a need for a method that in a simple way may represent the rotor loading by body forces without prior knowledge of the turbine. The aim of the present investigation is to develop a tool that, in a simple analytical way, may include axial and azimuthal body forces mimicking most common rotor loadings.

In the paper we show how a general and universal analytical body force model can be derived on the basis of a simple concept assuming that the rotor loading is determined by a constant circulation on the rotor, modified by tip and root corrections.

\section{The actuator disc model}

In this section we describe the basic flow equations and the new approach for modeling the body forces representing the loading on the rotor blades.

\subsection{Basic flow equations}

To represent the effect from the actuator disc, as well as the velocity profile of the atmospheric boundary layer and turbulence, in the Navier-Stokes equations, body forces are added to the momentum equation, as shown below,

$$
\frac{\partial \mathbf{u}}{\partial t}+(\mathbf{u} \cdot \nabla) \mathbf{u}=-\frac{1}{\rho} \nabla p+v\left(1+v_{t} / v\right) \nabla^{2} \mathbf{u}+\mathbf{f}_{\mathrm{AD}} / \rho+\mathbf{f}_{\mathrm{BL}} / \rho+\mathbf{f}_{\mathrm{T}} / \rho
$$

Where $\mathbf{u}=(u, v, w)$ is the velocity vector, $p$ is the pressure, $\rho$ is the density of air, and $v=\mu / \rho$ is the kinematic viscosity, with $\mu$ being the absolute viscosity, and $v_{t}$ is the eddy viscosity. The last three terms on 
the right hand side denote various body forces, representing, respectively, the rotor loading, $\mathbf{f}_{\mathrm{AD}}$, a prescribed boundary layer profile, $\mathbf{f}_{\mathrm{BL}}$, and the influence of turbulence, $\mathbf{f}_{\mathrm{T}}$.

The simulations are performed with the EllipSys3D code orignially developed at DTU by Michelsen [18]-[19] and Sørensen [20]. The code is a general purpose solver, based on a finite volume approach, which is formulated in primitive variables in a collocated grid arrangement. Furthermore, the code is parallized using MPI. The numerical model uses a blend of 10\% QUICK and 90\% fourth-order CDS for the convective terms, and a pure second-order CDS for the remaining terms. For pressure correction, the SIMPLE method is used. For more details regarding the numerical technique, we refer to Sørensen [20].

The three force terms appearing on the right hand side of the flow equations represent various ways of introducing kinematic properties through prescribed body forces. Besides the loading on the rotor disc, $\mathbf{f}_{\mathrm{AD}}$, which will be discussed later, the desired wind shear is introduced into the computations through the $\mathbf{f}_{\mathbf{B L}}$ term, using the prescribed boundary layer method described by Troldborg et al. [21]. Furthermore, in a first step, a syntethic atmospheric turbulence field is generated using the Mann model [22]-[23]. By adjusting the roughness length parameter, a desired turbulence intensity (Ti) level can be reached. In a second step, the turbulence field is recalculated into body forces and divided into a number of streamwise planes. These planes are inserted close to the inlet, upstream of the wind turbines, and introduced by using the $\mathbf{f}_{\mathrm{T}}$ term in eq. (1). The body forces need to be smoothly re-distributed to prevent numerical oscillations and to ensure that the integrated loading is conserved. As the disc appears as a one-dimensional line in the axisymmetric plane, a one-dimensional Gaussian filtering approach is applied to re-distribute the forces away from the disc. In the current model, each force element is filtered in the normal direction with a distance $d$ away from the disc according to the convolution,

$$
\mathbf{f}_{\text {res }}=\mathbf{f}_{\mathrm{AD}} \otimes \eta^{1 D}
$$

Here $\mathbf{f}_{\mathbf{A D}}$ is the resulting body force and the smearing function $\eta^{1 D}$ is computed as

$$
\eta^{1 D}(d)=\frac{1}{\sqrt{\pi} \varepsilon} \exp \left[-\left(\frac{d}{\varepsilon}\right)^{2}\right]
$$

For more information about the actuator disc concept and the smoothing technique, the reader is referred to the work of Sørensen and Shen [14] and Mikkelsen [24].

\subsection{Analytical body force model}

In the following subsection we describe in detail the derivation of the new analytical body force model. Applying the Bernoulli equation in a rotating frame of reference across the rotor plane, we get

$$
p^{+}+1 / 2 \rho\left(u^{2}+v^{2}+(\Omega r)^{2}\right)=p^{-}+1 / 2 \rho\left(u^{2}+v^{2}+\left(\Omega r+u_{\theta}\right)^{2}\right),
$$

where $\Omega$ is the angular velocity of the rotor, $u_{\theta}$ is the azimuthal velocity in the wake just behind the rotor, and $p$ is the pressure, with the sign denoting whether it is in front of or behind the rotor plane. Reducing the expression, we get the following equation for the pressure drop,

$$
\Delta p=\rho \Omega r u_{\theta}+1 / 2 \rho u_{\theta}^{2}
$$


From this plus Euler's turbine equation, we get the following two equations for the surface sources acting on the actuator disc representing the wind turbine:

$$
\begin{aligned}
& f_{z}=\rho u_{\theta}\left(\Omega r+1 / 2 u_{\theta}\right) \\
& f_{\theta}=\rho u_{D} u_{\theta}
\end{aligned},
$$

where $f_{z}$ and $f_{\theta}$ are the axial and azimuthal surface forces, respectively, and $u_{D}=u_{D}(r)$ denotes the axial velocity in the plane of the rotor. Making these equations dimensionless, we get

$$
\begin{aligned}
\frac{f_{z}}{\rho U_{0}^{2}} & =\frac{u_{\theta}}{U_{0}}\left(\frac{\Omega R}{U_{0}} \frac{r}{R}+1 / 2 \frac{u_{\theta}}{U_{0}}\right)=\frac{u_{\theta}}{U_{0}}\left(\lambda x+1 / 2 \frac{u_{\theta}}{U_{0}}\right), \\
\frac{f_{\theta}}{\rho U_{0}^{2}} & =\frac{u_{D}}{U_{0}} \frac{u_{\theta}}{U_{0}}
\end{aligned}
$$

where $\lambda \equiv \frac{\Omega R}{U_{0}}$ is the tip speed ratio and $x=r / R$, with $R$ denoting the radius of the rotor.

The model we are going to use for determining the body forces is an actuator disc with a constant circulation, corresponding to a Joukowsky rotor, modified with a tip correction, $F(r)$, and a root correction, $g(r)$. Hence, the azimuthal velocity distribution is given as

$$
u_{\theta}=\frac{\Gamma_{0}}{4 \pi r} \cdot g(r) \cdot F(r)
$$

where $\Gamma_{0}$ is the reference circulation, and the term $\frac{\Gamma_{0}}{4 \pi r}$ is the induction from an half-infinite straight vortex, corresponding to a root vortex.

As tip correction we employ the classical one by Prandtl (Betz [25]),

$$
F=\frac{2}{\pi} \arccos \left[\exp \left(-\frac{N_{b}}{2} \sqrt{1+\lambda^{2}} \cdot(1-x)\right)\right],
$$

where $N_{b}$ denotes the number of rotor blades.

To account for the influence of the hub and the inner non-lifiting part of the rotor, a vortex core of size $\delta$ is introduced, and an expression for the root correction is proposed as follows,

$$
g=1-\exp \left[-a\left(\frac{x}{\bar{\delta}}\right)^{b}\right]
$$


where $\bar{\delta}=\frac{\delta}{R}$ denotes the dimensionless radial distance to the point where the maximum azimuthal velocity is achieved. With the proposed model, $\bar{\delta}$ typically corresponds to the point where the lifting surface of the rotor starts. The expression for the correction $g$ is inspired by the work of Delery [26], who introduced a similar model for expressing the inner viscous core of a vortex. In his model, an exponent $b=2$ was employed and the corresponding coefficient was found to be $a=1.256$. In the general case, the relation between the constants $a$ and $b$ in eq. (10) is determined by differentiating eq. (8) to determine the maximum azimuthal velocity at $x=\bar{\delta}$. Since the tip correction approximately is unity at the root, we get the following implicit expression for determining the relationship between the coefficients $a$ and $b$,

$$
(a \cdot b+1) \exp (-a)=1 \text {. }
$$

In the following, we employ a value $b=4$, which then results in a value $a=2.335$. It should be noted that this largely is an empirical fit to the actual distribution of the azimuthal velocity at the inner non-lifting part of the rotor.

Introducing the dimensionless reference circulation as $q_{0}=\frac{\Gamma_{0}}{4 \pi R U_{0}}$, in dimensionless form, the azimuthal velocity is written as

$$
\frac{u_{\theta}}{U_{0}}=q_{0} \frac{g(x) F(x)}{x}
$$

and the dimensionless surface forces are written as

$$
\begin{aligned}
& \frac{f_{z}}{\rho U_{0}^{2}}=q_{0} \frac{g F}{x}\left(\lambda x+1 / 2 q_{0} \frac{g F}{x}\right) . \\
& \frac{f_{\theta}}{\rho U_{0}^{2}}=\frac{u_{D}}{U_{0}} q_{0} \frac{g F}{x}
\end{aligned}
$$

Assuming constant inflow conditions, the surface forces remain axisymmetric, and the thrust acting on the actuator disc is given by integration of the axial loading,

$$
T=\int_{A D} f_{z} d A=\int_{0}^{R} f_{z} 2 \pi r d r=2 \pi R^{2} \int_{0}^{1} x f_{z} d x
$$

The associated thrust coefficient is given as

$$
C_{T}=\frac{T}{1 / 2 \rho \pi R^{2} U_{0}^{2}}=\frac{4}{\rho U_{0}^{2}} \int_{0}^{1} x f_{z} d z=4 q_{0} \lambda \int_{0}^{1} g F x d x+2 q_{0}^{2} \int_{0}^{1} \frac{g^{2} F^{2}}{x} d x
$$

which can be written as 


$$
C_{T}=2 a_{1} q_{0}^{2}+4 \lambda a_{2} q_{0}
$$

with $a_{1}=\int_{0}^{1} \frac{g^{2} F^{2}}{x} d x$ and $a_{2}=\int_{0}^{1} g F x d x$.

With thrust coefficient $C_{T}$ and tip speed ratio $\lambda$ given, the dimensionless reference circulation is determined from eq. (16),

$$
q_{0}=\frac{\sqrt{16 \lambda^{2} a_{2}^{2}+8 a_{1} C_{T}}-4 \lambda a_{2}}{4 a_{1}} .
$$

Having determined the reference circulation from eq. (16), assuming a given thrust coefficient and tip speed ratio, the force distributions are determined from eq. (12). It should be noted that the axial force distribution is fixed, whereas the azimuthal force distribution depends on the local axial velocity in the rotor plane. This is obtained as a part of the computation and will hence vary during the simulations until a steady solution is obtained. The moment acting on the rotor is given by integration of the azimuthal force,

$$
M=\int_{A D} r f_{\theta} d A=2 \pi R^{3} \int_{0}^{1} x^{2} f_{\theta} d x .
$$

The power yield is given as

$$
P=M \Omega=2 \pi \Omega R^{3} \int_{0}^{1} x^{2} f_{\theta} d x
$$

with the resulting power coefficient given as

$$
C_{P}=\frac{M \Omega}{1 / 2 \rho \pi R^{2} U_{0}^{3}}=4 \lambda q_{0} \int_{0}^{1}\left(\frac{u_{D}}{U_{0}}\right) g F x d x
$$

\subsection{Upstream reference velocity and non-uniform inflow}

In a wind farm it is difficult determine the 'undisturbed' upstream wind speed, $U_{0}$, as the upstream flow is varying in space and time due to turbulence and wake effects from surrounding wind turbines. A way to determine $U_{0}$ is to compute an average axial velocity 2-3 diameters upstream of the turbine at hub height. However, it is not known if the velocity at this position corresponds to the 'undisturbed' upstream velocity, as the neighboring wind turbines influences the velocity field. Another technique is to employ one-dimensional momentum theory to derive an approximate expression expressing the relationship between the upstream velocity and the average velocity at the rotor disc. Employing a control volume which encompasses the rotor and which follows the stream surface from far upstream to far downstream, the following expression is obtained, 


$$
C_{T}=4 \frac{\bar{u}_{D}}{U_{0, r e f}}\left[1-\frac{\bar{u}_{D}}{U_{0, r e f}}\right],
$$

where $\bar{u}_{D}=\frac{1}{A} \int_{A D} u_{D} d A$ denotes the average axial disc velocity and $U_{0, r e f}$ is the upstream 'undisturbed' reference velocity. Re-arranging eq. (20), the upstream reference velocity is given as

$$
U_{0, r e f}=\frac{2 \bar{u}_{D}}{1+\sqrt{1-C_{T}}} .
$$

In general the inflow is non-axisymmetric, due to wind shear, wake effects and possible yaw misalignment, and unsteady due to atmospheric turbulence. This implies that $U_{0}=U_{0}(x, \theta, t)$, and hence that $u_{D}=u_{D}(x, \theta, t)$. Assuming that $\frac{U_{0}}{U_{0 . r e f}}=\frac{u_{D}}{\bar{u}_{D}}$, we get $U_{0}=\frac{2 u_{D}}{1+\sqrt{1-C_{T}}}$. In the CFD simulations, the rotor is discretized using a local polar grid. For each time step and at each node point of this grid, $u_{D, i}=u_{D, i}(x, \theta, t)$ is extracted and used to calculate the local surface forces using eqs. (11) and (12). Thus, generalizing eq. (21) and inserting it into eq. (12), at each node point, $i$, the local forces are computed as

$$
\begin{aligned}
& \Delta F_{z, i}=4 \rho q_{0} \frac{g F}{x}\left(\lambda x+1 / 2 q_{0} \frac{g F}{x}\right) \frac{u_{D, i}^{2} \Delta A_{i}}{\left(1+\sqrt{1-C_{T}}\right)^{2}}, \\
& \Delta F_{\theta, i}=2 \rho q_{0} \frac{g F}{x} \frac{u_{D, i}^{2} \Delta A_{i}}{\left(1+\sqrt{1-C_{T}}\right)}
\end{aligned}
$$

where $\Delta F_{z, i}$ and $\Delta F_{\theta, i}$ denote the axial force and tangential force components, respectively, acting on surface area $\Delta A_{i}$. In the actuator disc model, these terms form the basis for the body forces representing the rotor loading in the Navier-Stokes equations, eq. (1). The actual implementation of the terms in general depends on the numerical stencil used for solving eq. (1) and the filter in the smearing function, eqs. (2) and (3).

In the case where computations are being carried out without prior knowledge of the exact size of the rotor, the following expression can be utilized to determine the relationship between rotor size and generator size,

$$
P_{\text {rated }}=1 / 2 \rho \pi R^{2} U_{\text {rated }}^{3} C_{P, \text { design }},
$$

where $U_{\text {rated }}$ is the rated wind speed, i.e. the wind speed at which the rotor reaches the rated generator power, $P_{\text {rated }}$. From eq. (23), we get the following expression for determining the rotor radius, 


$$
R=\sqrt{\frac{2 P_{\text {rated }}}{\pi \rho U_{\text {rated }}^{3} C_{P, \text { design }}}}
$$

A typical value for the design power coefficient is 0.5 , the rated power is a question of the choice of the most appropriate wind turbine on the marked at the given time, and the rated wind speed may be varied according to the local geostrophic wind speed and roughness parameters. With this expression, together with the generic force distributions, eq. (22), it is in principle not required to have any apriori knowledge of the actual wind turbine to be analysed.

\section{Results}

In the following, results from the analytical model will be presented and compared with results from CFD actuator disc simulations using actual rotor geometries and airfoil data.

\subsection{Analytical load distributions}

To give an idea of how the velocity and load distributions are distributed as function of the parameters introduced in section 2.1, various plots will be shown in the following. To simplify the analysis, in the following we assume that $\bar{\delta}=0.1$.

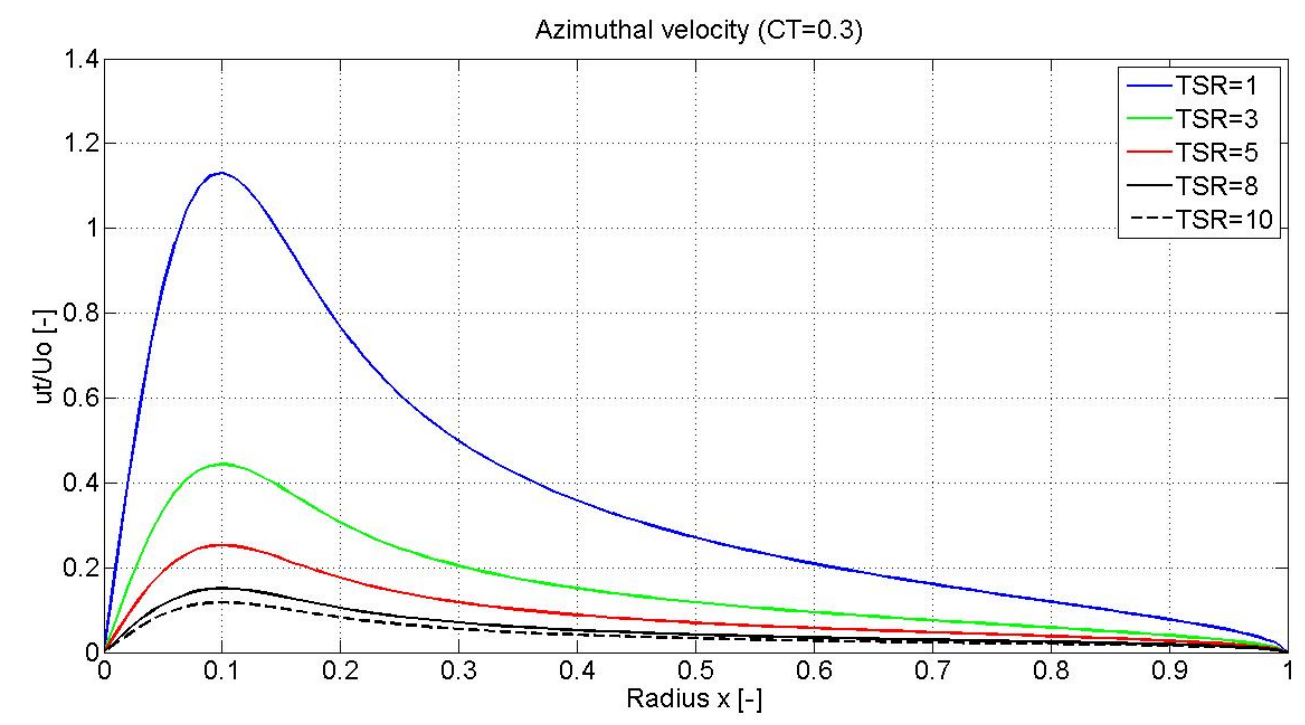

Figure 2. Azimuthal velocity distribution along the radius for different tip speed ratios at $C_{T}=0.3$.

In Figs. 2 and 3 the azimuthal velocity distribution (eq. 11) is depicted for different tip speed ratios at a thrust coefficient of 0.3 and 0.9 , respectively. Here it is seen that that the expression for the velocity distribution, eq. (11), exactly gives that the maximum velocity is obtained at $x=\bar{\delta}$, corresponding to the core radius of the root vortex. It should be noted that the azimuthal force distribution, except for the mass flux (see eqs. 6 or 7), is idential to the azimuthal velocity. Thus, except for a scaling, the azimuthal force distribution has the same shape as the azimuthal velocity distribution. 


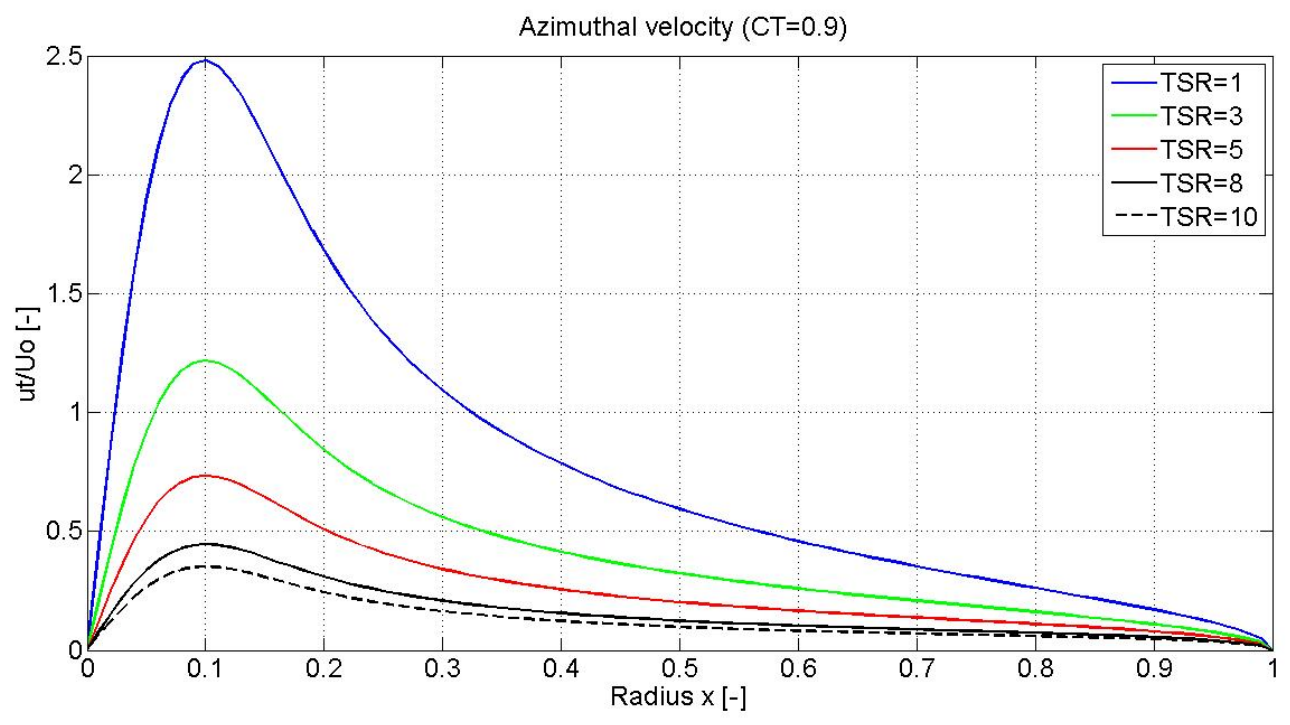

Figure 3. Azimuthal velocity distribution along the radius for different tip speed ratios at $C_{T}=0.9$.

The corresponding axial force distributions (eq. 12) are shown in Figs. 4 and 5, where it can be seen that the axial force distributions tend to a nearly constant value for tip speed ratios greater than 5 . This implies that, except for the root and tip part, a constant axial loading for most practical cases represents the physics of a constant circulation quite well. In Fig. 6 we depict the reference circulation, $q_{0}$, as a function of tip speed ratio for different thrust coefficients. In Fig. 7 the circulation is divided with the thrust coefficient, showing that the circulation scales with the thrust coefficient at moderate and high tip speed ratios.

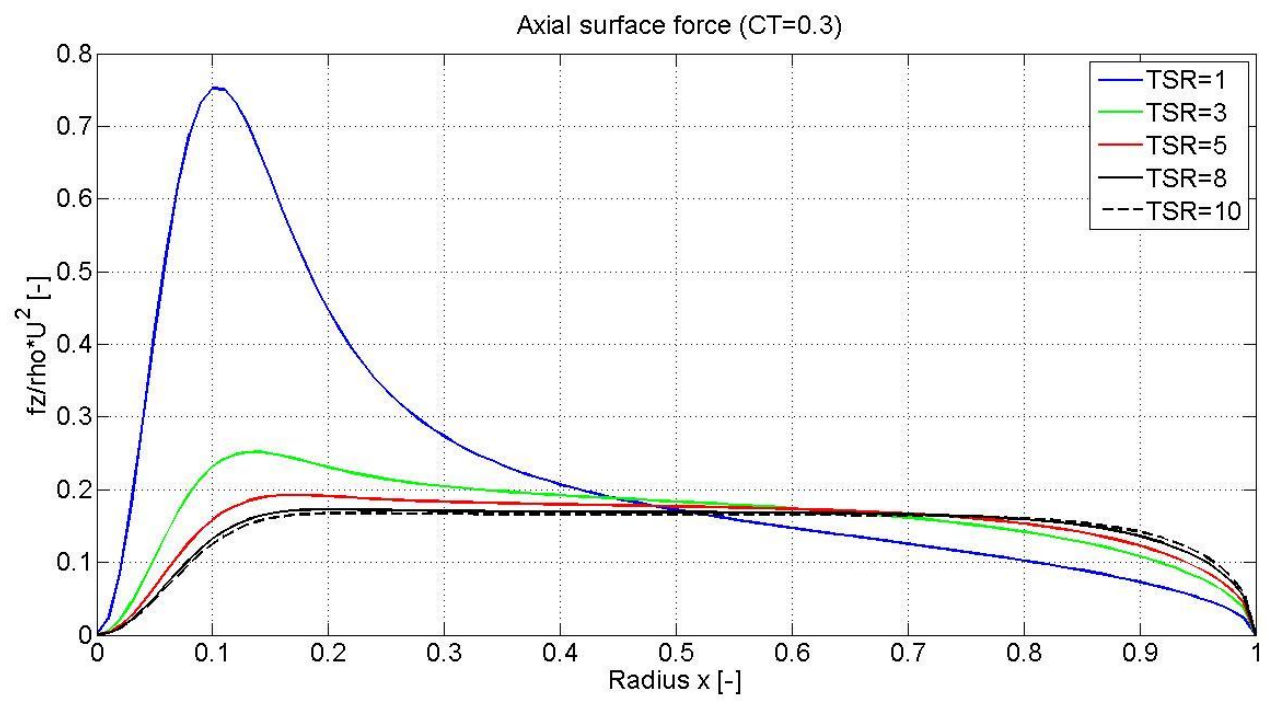

Figure 4. Axial force distribution along the radius for different tip speed ratios at $C_{T}=0.3$. 


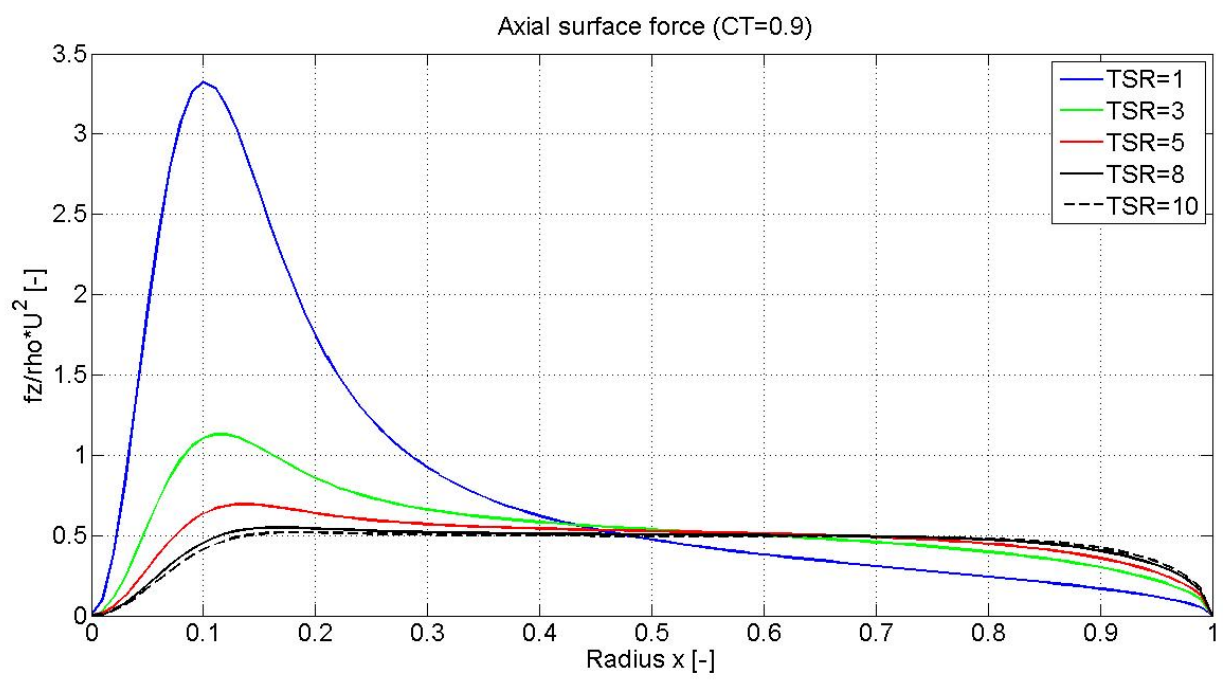

Figure 5. Axial force distribution along the radius for different tip speed ratios at $C_{T}=0.9$.

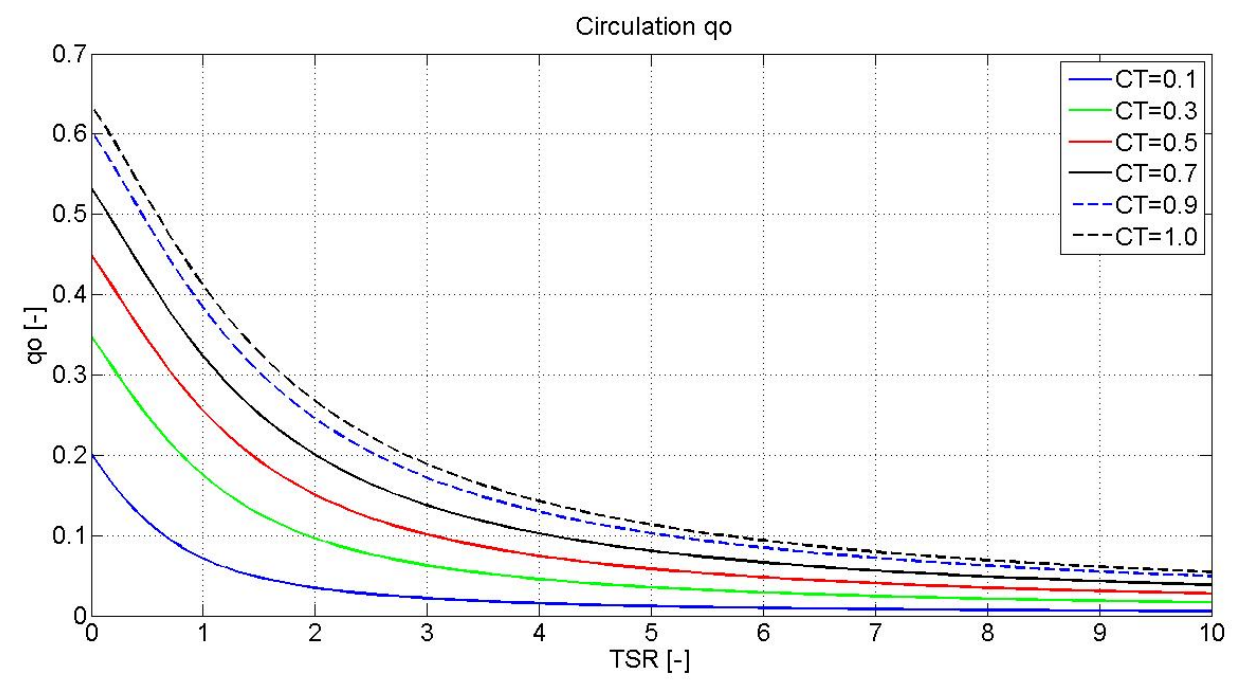

Figure 6. Reference circulation as a function of tip speed ratio for different thrust coefficients. 


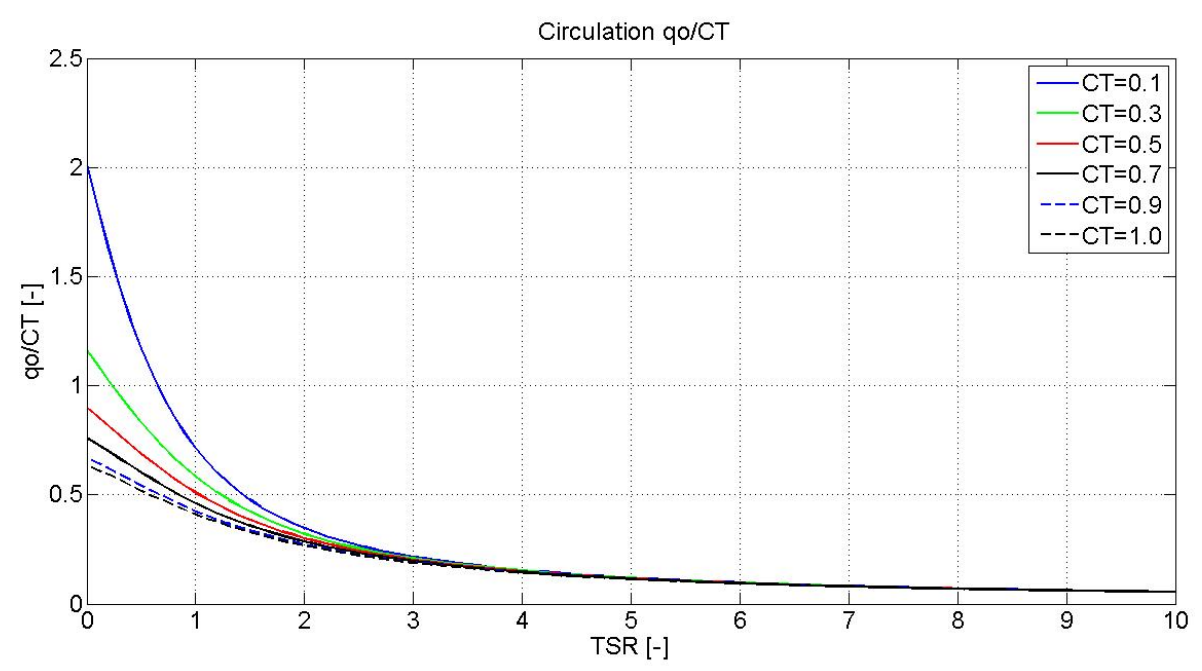

Figure 7. Reference circulation $q o / C_{T}$ as a function of tip speed ratio for different thrust coefficients.

\subsection{The Tjaereborg wind turbine}

In the following we validate the analytical model using numerical results obtained from the CFD code described in section 2.1 for the Tjaereborg wind turbine. The validation is based on comparing results using the analytical model with rotor radius, tip speed ratio and thrust coefficient as the only input, with results using actuator disc computations based on the full rotor geometry, i.e. chord-, twist-, and thickness-distributions, and measured airfoil data. The 2 MW Tjaereborg wind turbine, which was in operation in the period $1987-2001$, was developed in a cooperation between the Danish Ministry of Energy and the electric utilities as a part of a joint programme directed towards the development of large wind turbines. The turbine is a 3-bladed, horizontal axis turbine with a rotor diameter of $61.1 \mathrm{~m}$. The blade blade length is $29 \mathrm{~m}$ and the hub height is $60 \mathrm{~m}$. The blade sections consist of NACA 44xx airfoils with a chord length of $0.9 \mathrm{~m}$ at the tip and a linear increase from this of $0.1 \mathrm{~m}$ per span wise unit length. The rotor is linearly twisted 1 degree per $3 \mathrm{~m}$ and the turbine was operated with a constant pitch angle equal to zero for wind velocities up to about $12 \mathrm{~m} / \mathrm{s}$, after which the pitch angle is regulated to maintain a constant electrical power output at $2 \mathrm{MW}$. At rated power the rotor was operated at a nominal speed $22.36 \mathrm{rpm}$. Further technical details can be found in Friis [27].

\subsubsection{Single turbine in uniform inflow}

The first test was performed using a single wind turbine in a uniform inflow as reference and the analytical model both with rotation and without rotation. The latter was obtained simply be setting $f_{\theta}$ equal to zero, with the purpose of investigating the impact of the swirl flow induced in the wake by the action of $f_{\theta}$. In the plots the results obtained from the analytical model are referred to as 'generic'. The inflow is uniform with a velocity of $8 \mathrm{~m} / \mathrm{s}$ (to ensure that we are in the "middle" of the optimal operation regime). Three different turbulence levels were considered; $\mathrm{Ti}=0 \%, 5 \%$ and $10 \%$, respectively. The turbulence was determined in an empty domain at the location where the turbine was later imposed (see Fig. 8). First, the reference turbine was simulated for all Ti-levels using a PI controller ensuring that the rotor responded to the condition it was operating in. The generic rotor was then activated using a thrust coefficient $\mathrm{C}_{\mathrm{T}}=0.87$ and a tip speed ratio of 8 (based on the reference simulations). The root correction was used with a 'viscous' core size $\bar{\delta}=0.23$, corresponding to the relative radius of the inner non-lifting part of the rotor. 


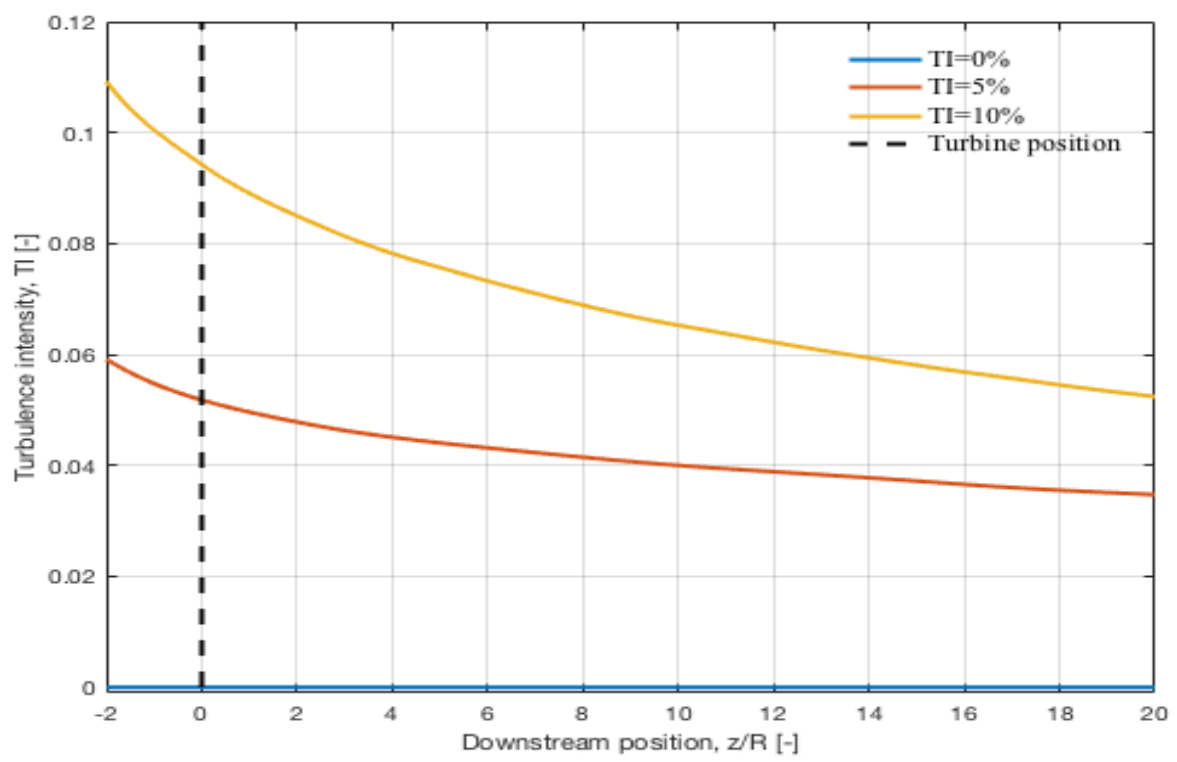

Figure 8. Determination of Ti level in the empty domain.

In Fig. 9, analytical load distributions are compared to computed distributions. As seen from the figures there is a remarkably good agreement between the analytical and the computed distributions. It is somewhat surprising that the agreement is that good, as the rotor was originally designed using the design methodology of Glauert (1935), which is based on momentum theory and therefore different from the assumption of constant
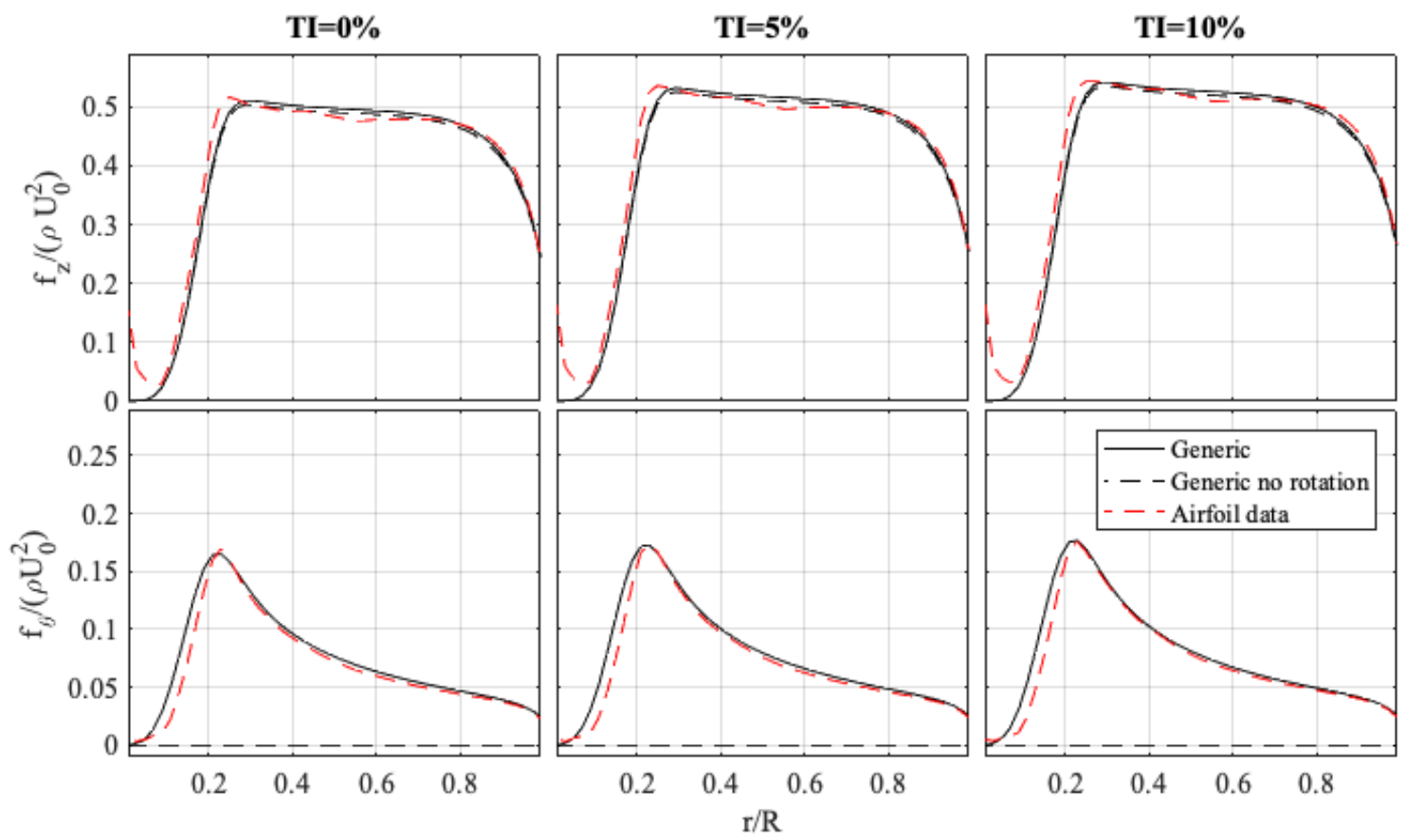

Figure 9. Comparison between loadings computed with the analytical (generic) model and loadings using airfoil data. The upper curves show axial surface forces and the lower curves show azimuthal surface forces. Red dashed lines: reference turbine using airfoil data; solid lines: generic; dashed lines: generic, no rotation. 
circulation used in the derivation of the analytical form. In spite of this, the analytical model is seen to follow very closely the distribution of the computed results, which, in contrast to the analytical method, demands detailed knowledge of the actual geometry as well as access to tabulated airfoil data.

To study the effect of using only the axial force component as compared to using a combined axial/azimuthal distribution, computations were carried out both with and without the azimuthal surface force, $f_{\theta}$. The results of this are depicted in Figs. $10-11$, which show the velocity profiles at different axial positions downstream the rotor and for different turbulence intensities. By comparing distributions of the axial velocity components (Fig. 10), it is seen that including the azimuthal velocity distribution indeed has an influence on the resulting axial flow distribution. At zero inflow turbulence, neglecting the azimuthal force component, and hence ignoring the azimutal velocities in the wake, causes a later development of the wake. This is in particular visible at $z=17 R$, where traces of the tip vortices are still visible where the wake center is seen to be less developed.
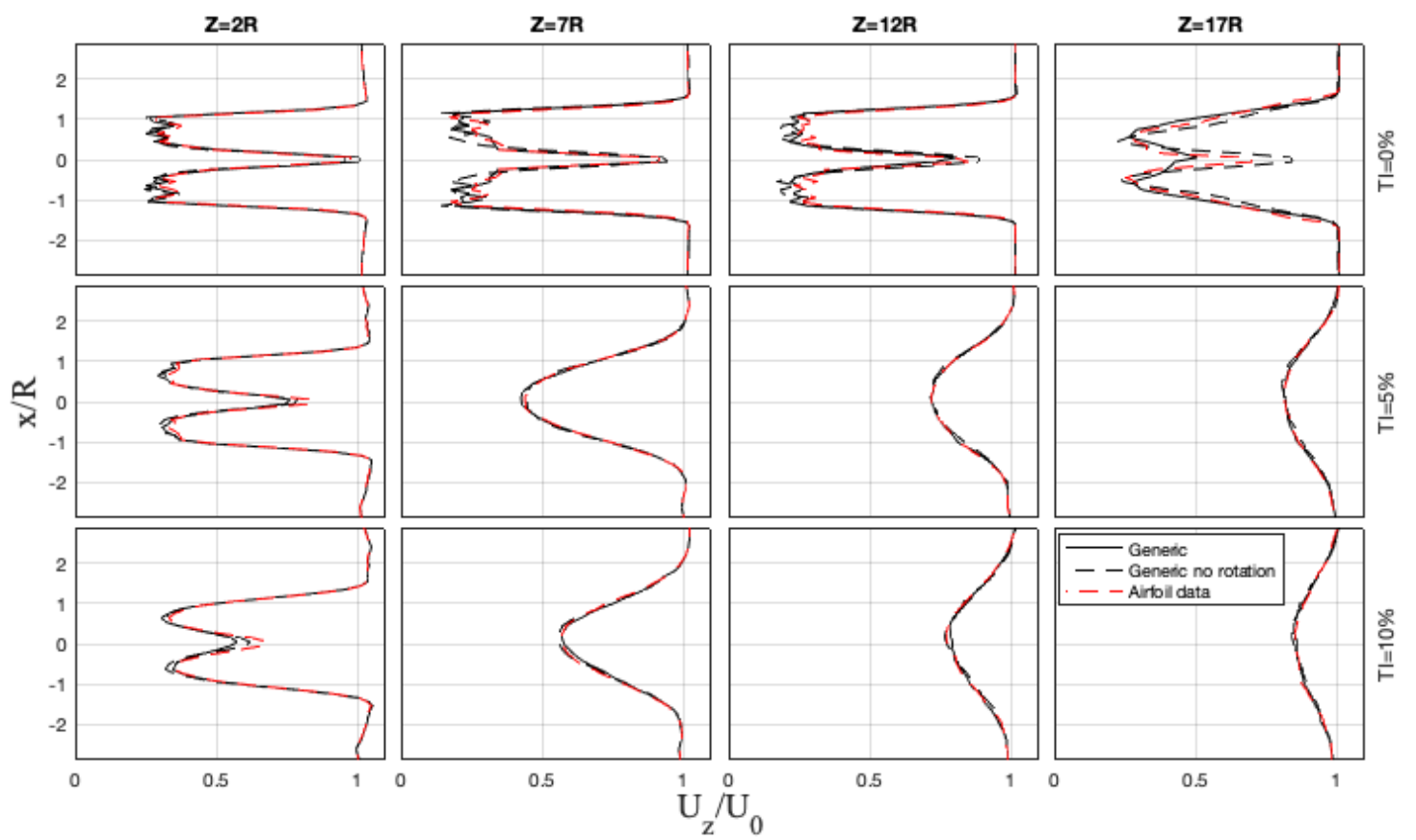

Figure 10. Distributions of axial velocity at various downstream locations. Upper plots: $\mathrm{Ti}=0 \%$; Middle plots: $\mathrm{Ti}=5 \%$; Lower plots: $\mathrm{Ti}=10 \%$. Red dashed lines: reference turbine using airfoil data; solid lines: generic; dashed lines: generic no rotation.

The resulting azimuthal velocity distributions are shown and compared in Fig. 11, where an excellent agreement is seen to exist between the distributions based on computations using the full geometry and airfoil data and those computed using the generic form of the surface forces. Naturally, except for some minor perturbations due to turbulent mixing, the azimuthal velocity component is zero when the azimuthal body forces are ignored. 

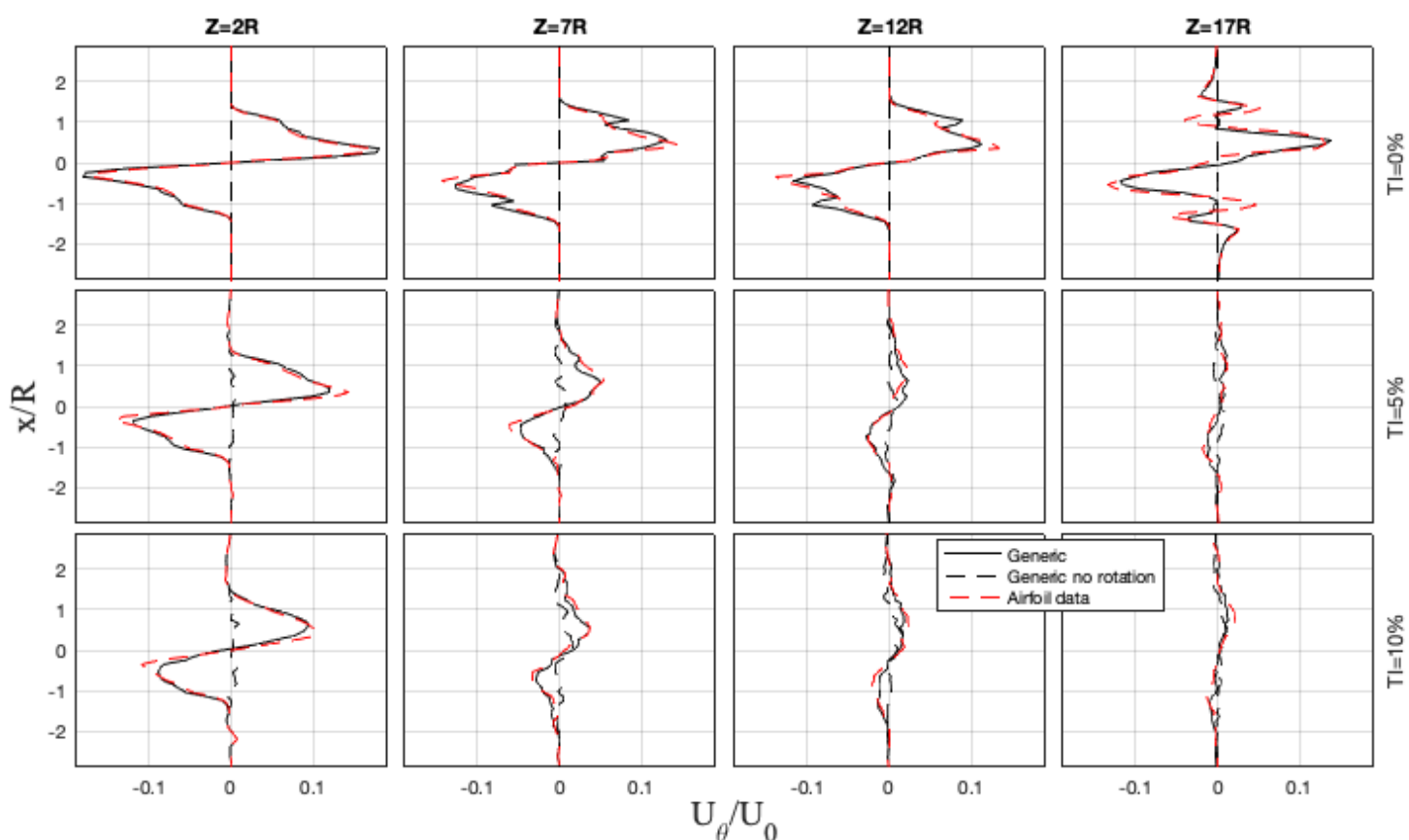

Figure 11. Distributions of azimuthal velocity at various downstream locations. Upper plots: $\mathrm{Ti}=0 \%$; Middle plots: $\mathrm{Ti}=5 \%$; Lower plots: $\mathrm{Ti}=10 \%$. Red dashed lines: reference turbine; solid lines: generic; dashed lines: generic no rotation.

\subsubsection{Two turbines in a row in uniform inflow}

To test the analytical rotor model for wind turbine clusters or turbines located in wind farms, the same case as before is now simulated using two turbines located along a common line in the wind direction. All settings are the same as for the case with a single turbine, and the second turbine is located 10 radii downstream of the first turbine. The analytical load distributions are given in Figs. 12 - 13 andcompared to the computed distributions for different turbulence intensities. The comparisons again show a very good agreement between the reference turbine using airfoil data and the analytical model, showing that the analytical form is valid for wind farm simulations. It also demonstrates that eq. (21) with good accuracy can be used to determine the correct reference velocity for the inflow to the second wind turbine. It is furthermore seen that the turbulence intensity has a big impact on the resulting force distributions of the second turbine. 


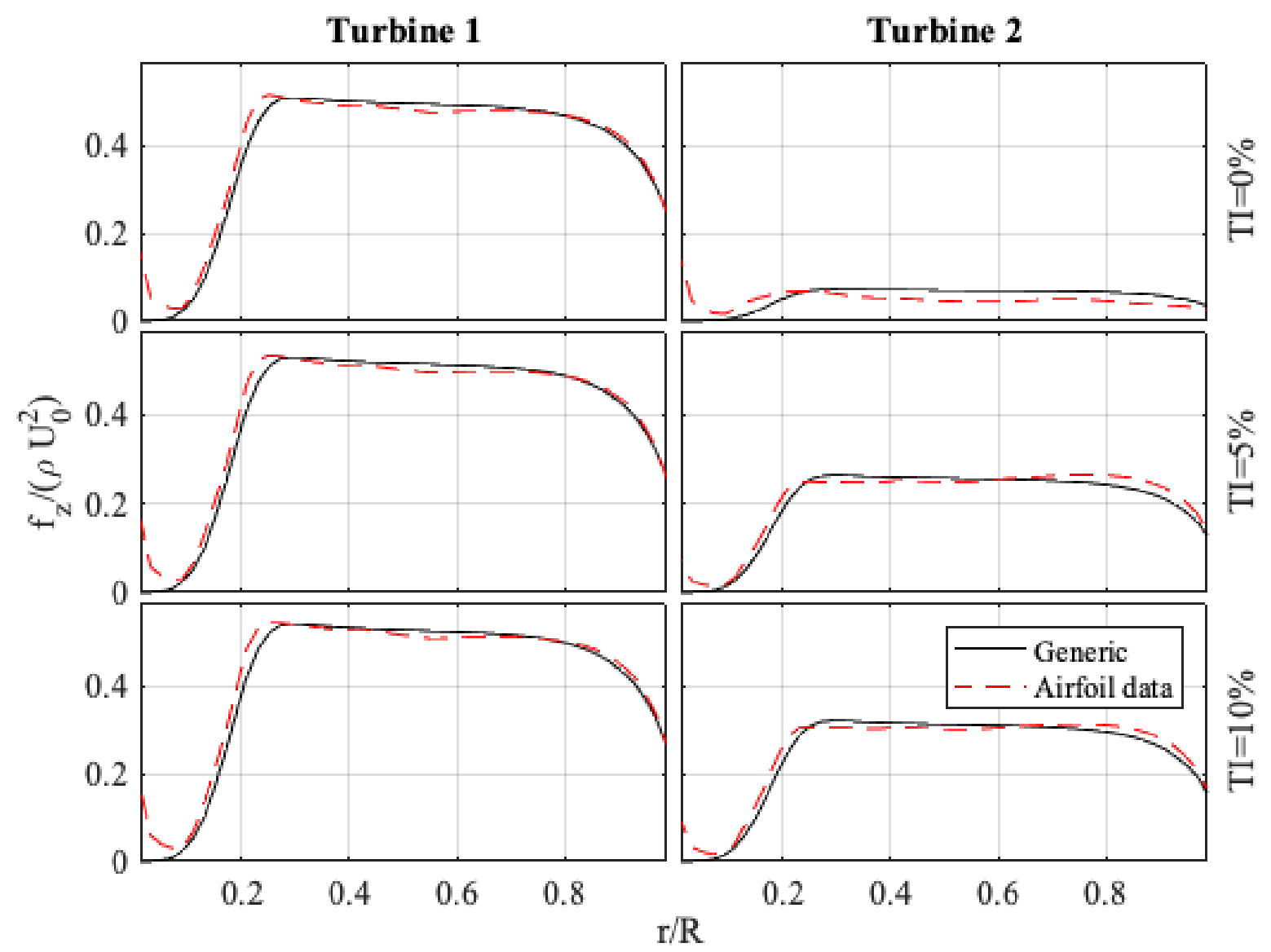

Figure 12. Comparison between axial surface forces computed with the analytical (generic) model and the reference model using airfoil data at different turbulence intensities. Left curves: upstream turbine; right curves: downstream turbine. Red dashed lines: reference turbine using airfoil data; solid lines: generic. 
Turbine 1

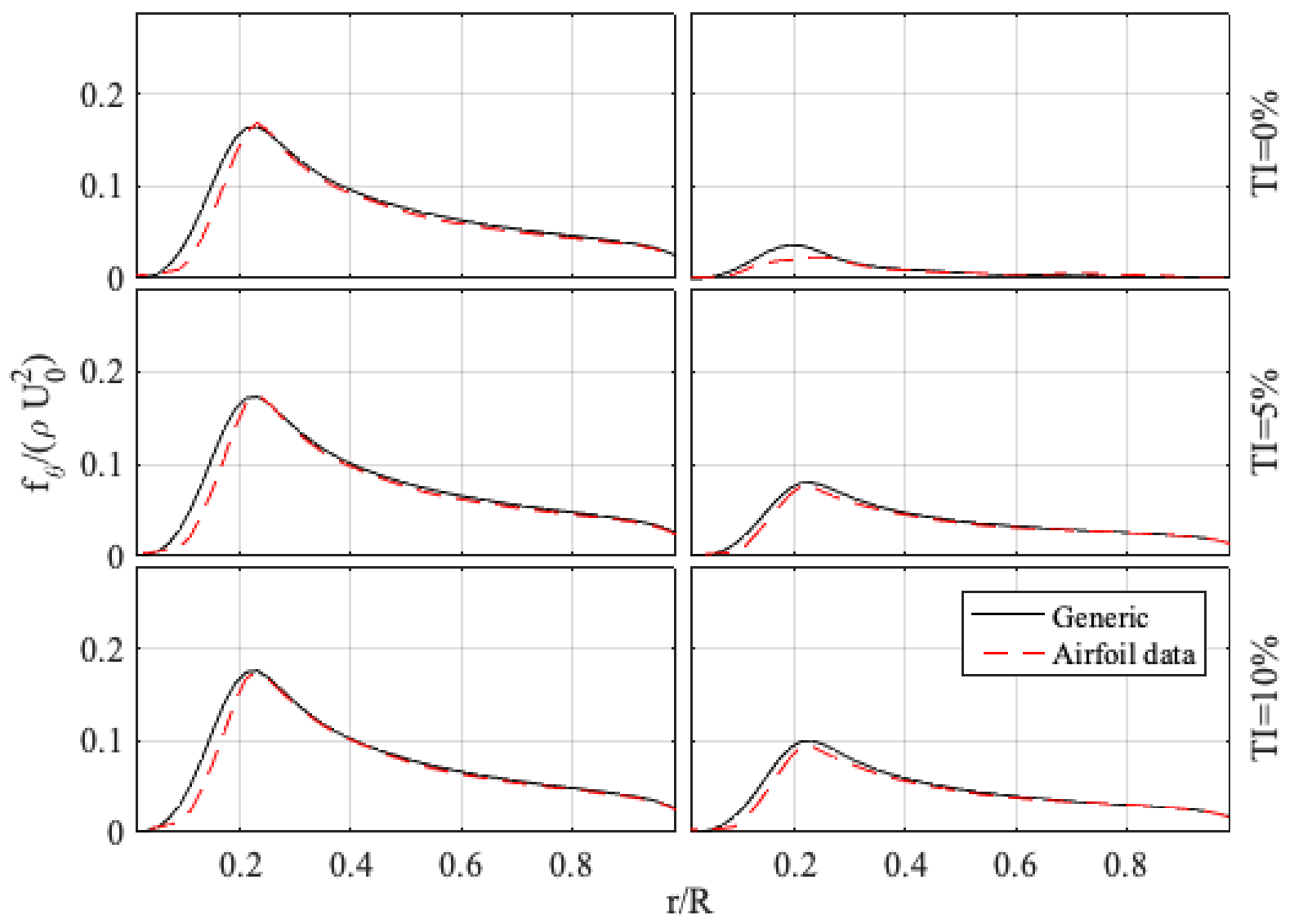

Figure 13. Comparison between azimuthal surface forces computed with the analytical (generic) model and the reference model using airfoil data at different turbulence intensities. Left curves: upstream turbine; right

curves: downstream turbine. Red dashed lines: reference turbine using airfoil data; solid lines: generic turbine.

\subsubsection{Two turbines in a row in sheared inflow}

To increase the complexity, we now consider two turbines located along a common axial line subjected to an imposed shear flow. This case has the same settings as the one above, with, however, only one Ti level (8\%). The thrust coefficeint was reevaluated, and found still to be equal to 0.87 in this case. The shear profile was defined using a power law with a shear exponent of 0.14 , and implemented using the prescribed boundary layer technique described in [21]. The averaged analytical load distributions are given in Figs. 14 and again compared to the computed averaged distributions for the two wind turbines. The comparisons again show a very good agreement between the loadings for the reference turbine using airfoil data and the generic turbine using the analytical model. 
Turbine 1

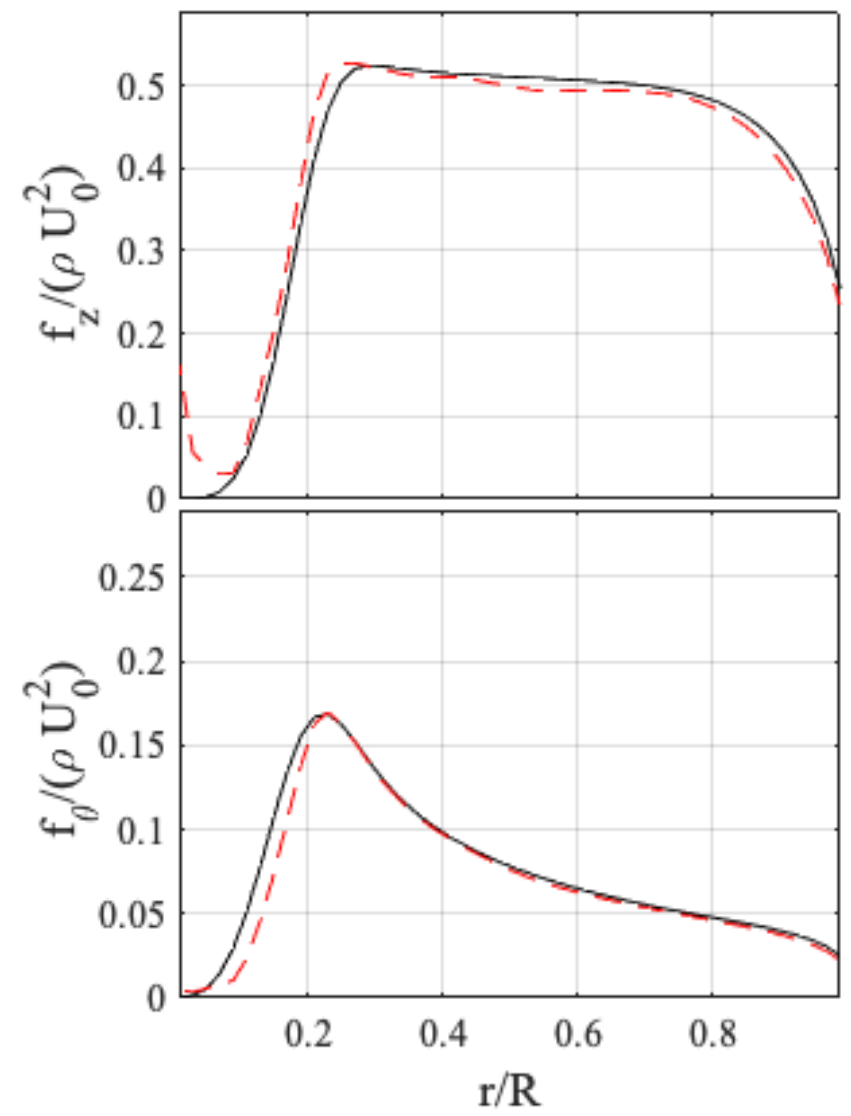

Turbine 2

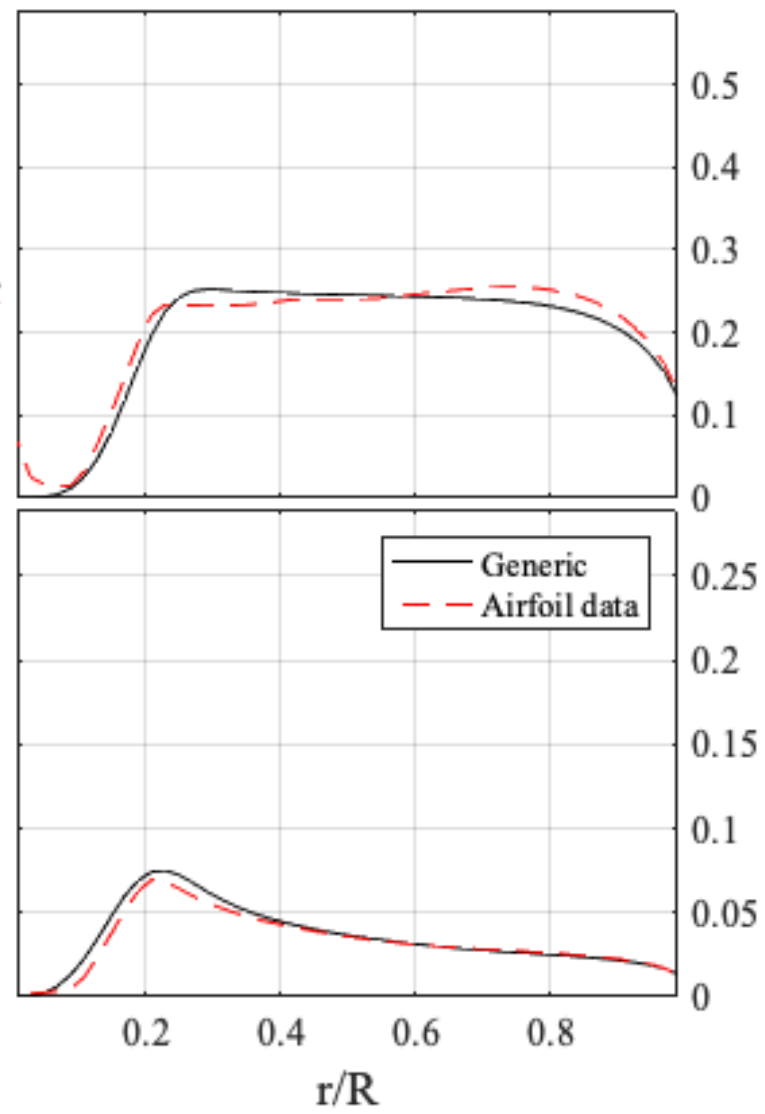

Figure 14. Comparison between loadings computed with the analytical (generic) model and loadings using airfoil data. The upper curves show averaged axial surface forces and the lower curves averaged azimuthal surface forces, with left curves showing distributions for the upstream turbine and the right curves for the downstream turbine. Red dashed lines: reference turbine using airfoil data; solid lines: generic turbine.

Resulting axial velocity distributions in a horizontal and a vertical midplane are shown and compared in Fig. 15, where an excellent agreement is seen to exist between the distributions based on computations using the reference turbine with airfoil data and those computed using the generic form using the analytical model of the surface forces. Similar distributions for the azimuthal velocity component are shown in Fig. 16, which again displays an excellent agreement between computations using the reference turbine with airfoil data and those computed using the generic form of the surface forces. 

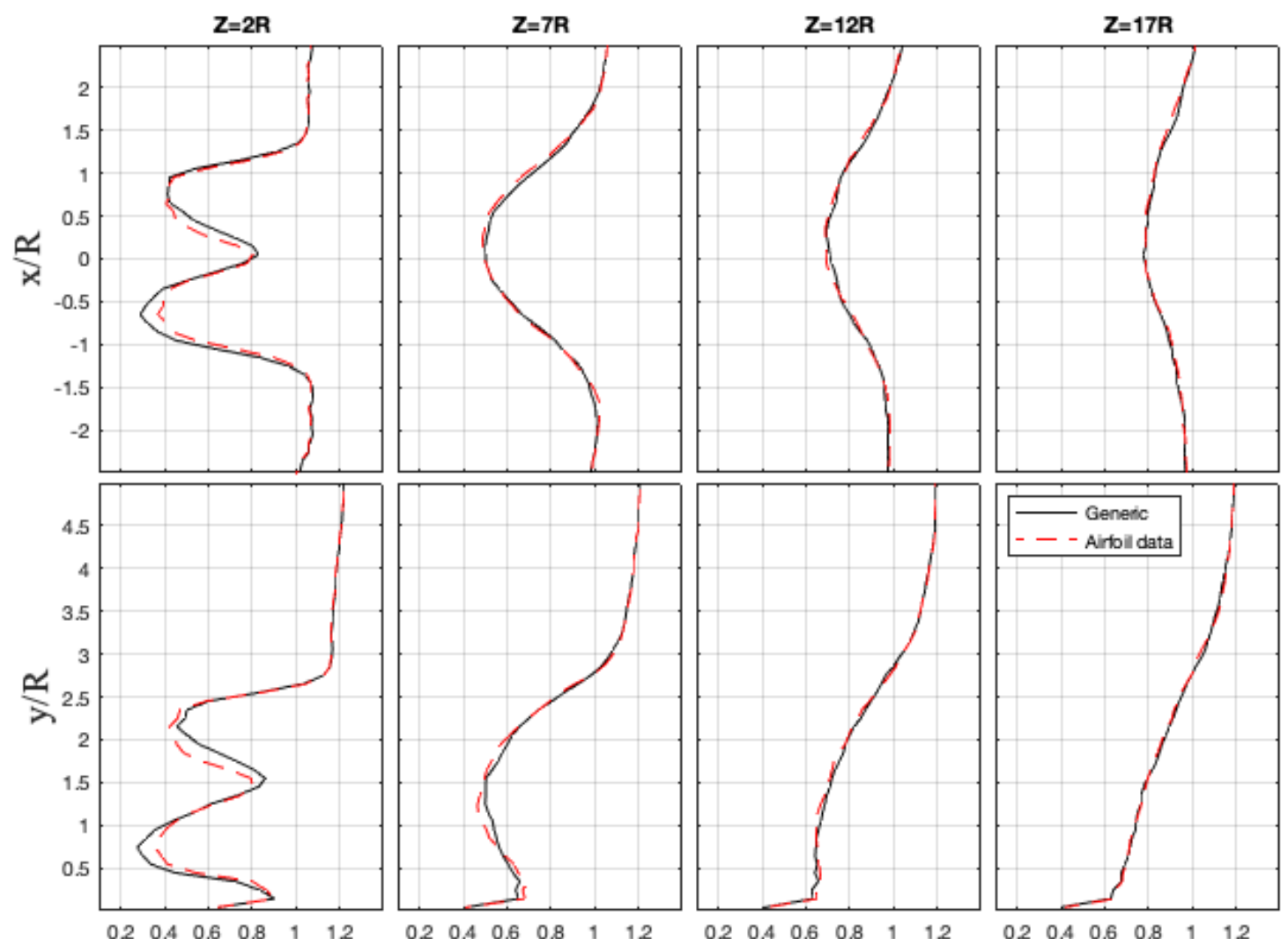

$\mathrm{U}_{\mathrm{z}} / \mathrm{U}_{0}$

Figure 15. Distributions of axial velocity at various downstream locations. Upper curves: horizontal midplane; lower curves: vertical midplane. Red dashed lines: reference turbine; solid lines: generic turbine. 

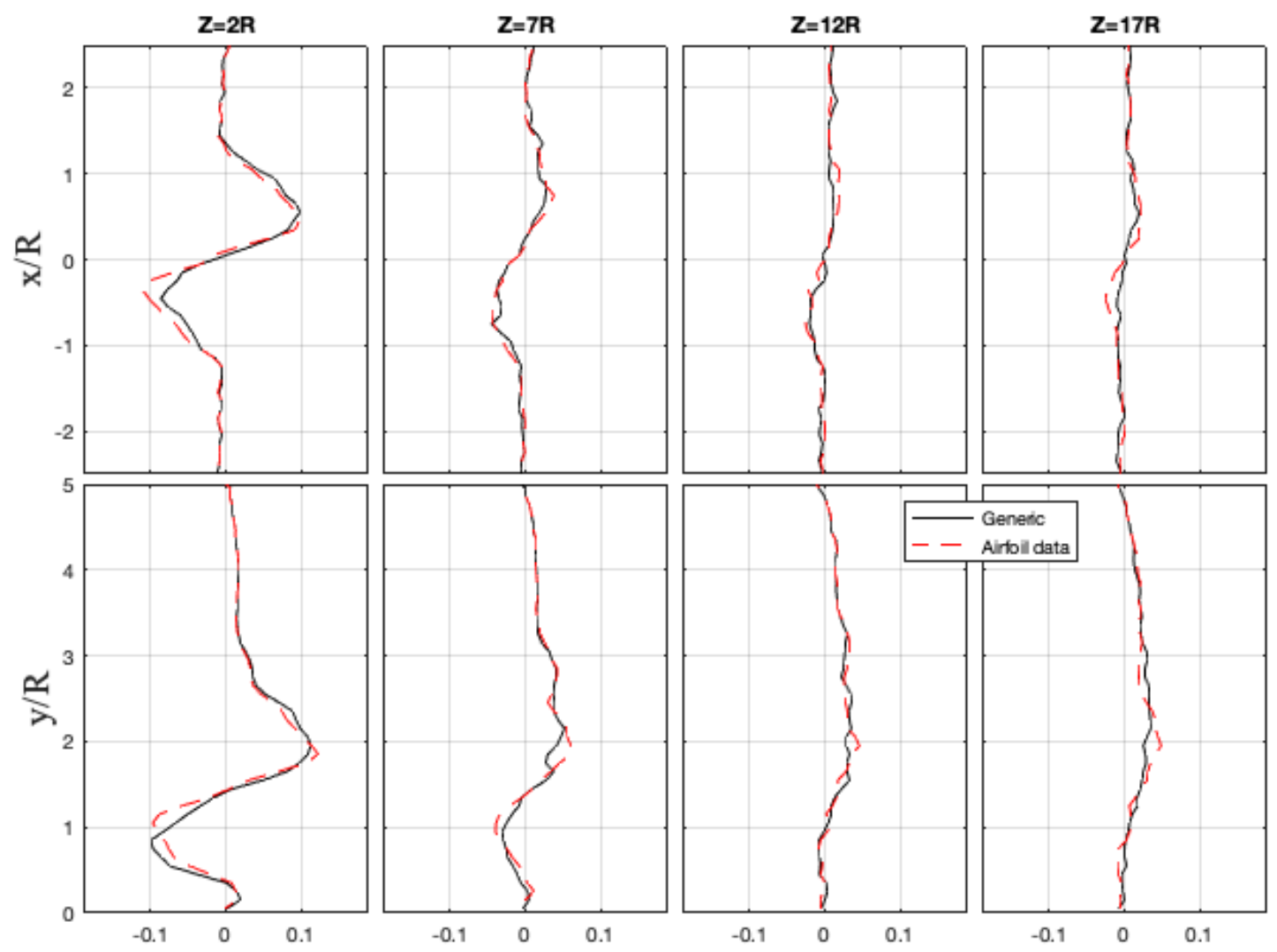

$\mathrm{U}_{\theta} / \mathrm{U}_{0}$

Figure 16. Distributions of azimuthal velocity at various downstream locations. Upper curves: horizontal midplane; lower curves: vertical mid plane. Read dashed lines: reference turbine; solid lines: generic turbine.

\subsection{The DTU 10MW reference wind turbine}

In the following we show results using the analytical model for the DTU 10MW reference wind turbine and again compare the outcome to results using full actuator disc computations based on measured airfoil data. The DTU 10MW reference wind turbine is a virtual wind turbine designed by DTU Wind Energy [28]. Inspired by the NREL $5 \mathrm{MW}$ wind turbine, it was designed to provide a new reference rotor for code validation and testing that was adapted to bigger upcoming turbine sizes. The turbine has a rotor diameter of $178 \mathrm{~m}$ and a hub height of $116 \mathrm{~m}$. All blade sections are based on the FFA-W3 airfoil series. In constrast to the Tjaereborg wind turbine, the aerodynamic design of the DTU reference wind turbine was highly constrained by secondary factors, such as structural properties and public availability of airfoil data. It therefore deviates more from an optimal rotor design than the Tjareborg wind turbine and thereby constitutes a more severe testcase regarding the universality of the proposed model. The CFD simulations were set up similarily to those of the Tjaereborg wind turbine. However, in the DTU wind turbine case, the thrust coefficient is set to 0.80 , the tip speed ratio to 8.1 and radius of the root core to 0.3 of the rotor radius. Furthermore, the computations were carried out with the rotor located in an atmospheric boundary layer with shear exponent 0.14 and a turbulence intensity $\mathrm{Ti}=8 \%$. 
The averaged load distributions for the generic rotor using the analytical model are in Fig. 17 compared to computed averaged distributions for the reference turbine using airfoil data. The comparisons show a very good agreement on the outer $60 \%$ of the rotor radius. On the inner $40 \%$ of the rotor, however, the comparison is less convincing, simply because the rotor design deviates significantly from the one utilized for the design of an overall aerodynamically optimal rotor. For example, it is observed that the geometry of the reference rotor results in a local maximum load value at $\mathrm{r} / \mathrm{R}=0.33$. Obviously, such a behavior cannot be captured by a simple analytical model assuming a constant circulation, corrected for root and tip effects. In spite of this, the resulting axial velocity distributions, shown in Fig. 18, exhibit an excellent agreement between the distributions based on computations using the reference turbine with airfoil data and those computed using the generic form of the surface forces.

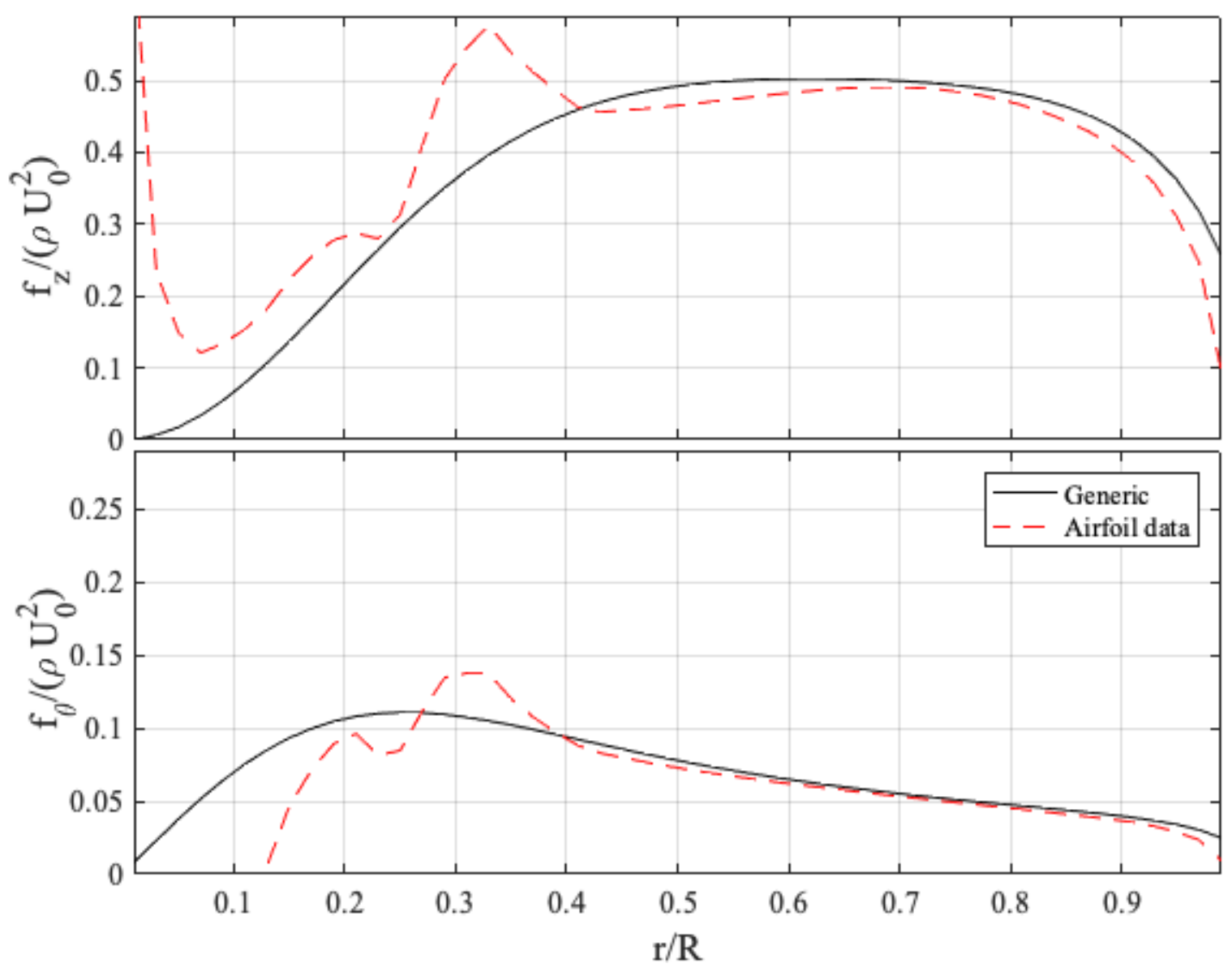

Figure 17. Comparison between loadings computed with the analytical (generic) model and loadings using airfoil data for the DTU 10MW reference turbine. The upper curves show averaged axial surface forces and the lower curves averaged azimuthal surface forces. Red dashed lines: reference turbine using airfoil data; solid lines: generic turbine. 

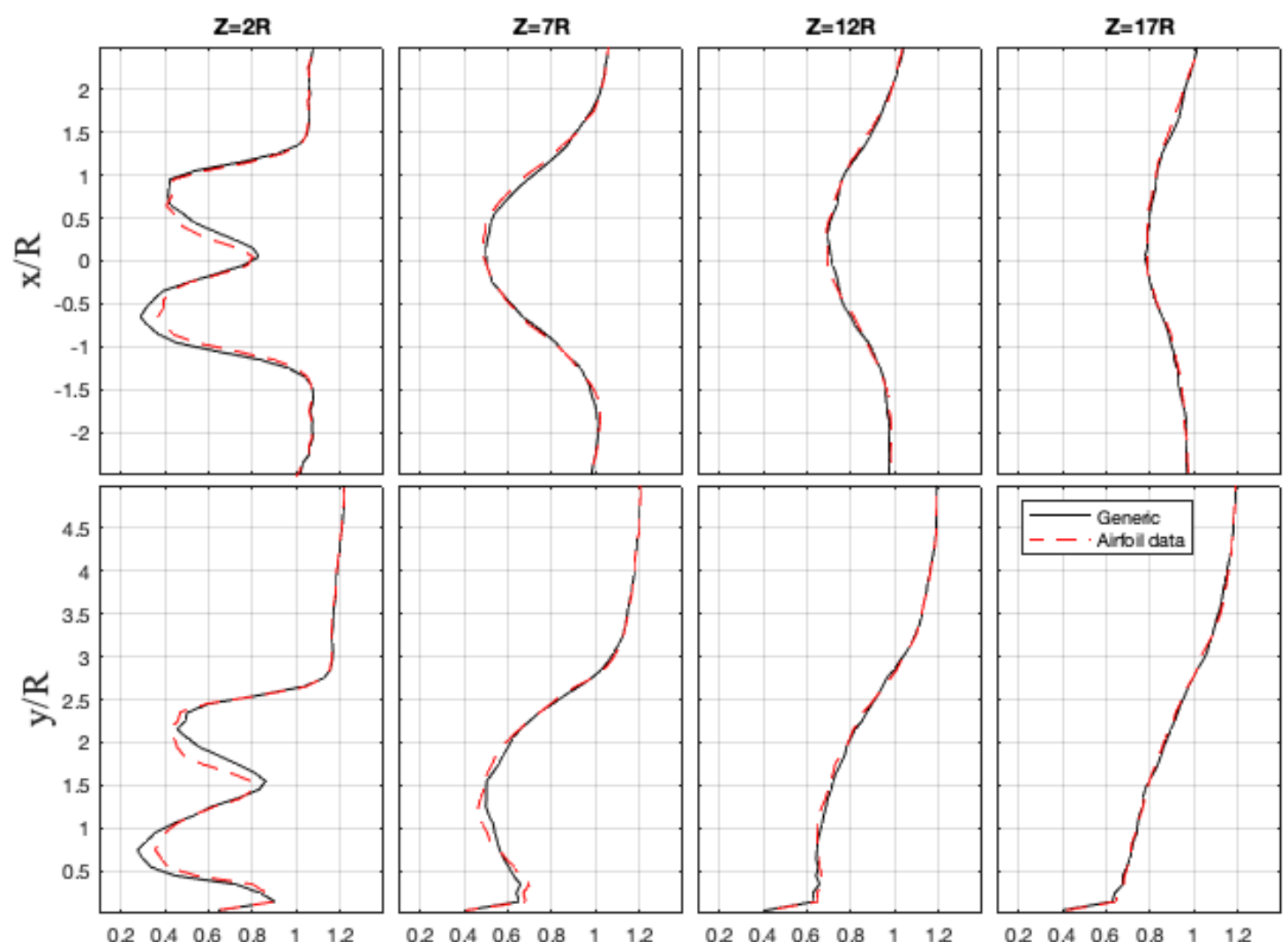

$$
\mathrm{U}_{\mathrm{z}} / \mathrm{U}_{0}
$$

Figure 18. Distributions of axial velocity at various downstream locations of the 10MW DTU reference turbine. Upper curves: horizontal midplane; upper curves: vertical midplane. Red dashed lines: reference turbine; solid lines: generic turbine.

\section{Conclusions}

In the paper an analytical model for representing body forces in a numerical actuator disc model of a wind turbine was developed. The main idea behind the model is the assumption that the rotor disc is subject to a constant circulation modified for tip and root effects. The derivation of the model is shown in detail, resulting in simple analytical expressions for the axial and the tangential force distributions. The advantage of the developed expressions are that they only depend on global parameters, such as rotor radius, tip speed ratio, and thrust coefficient. This can be further reduced to only depend on rated wind speed and nameplate capacity. Furthermore, the model is generalized to be be utilized for all kinds of inflow, including wind shear and turbulence, and for wind turbines subject to wake effects, e.g. from neighboring wind turbines in wind farms.

To validate the analytical model, results were compared to numerically generated results using detailed information regarding geometry and airfoil data for the $2 \mathrm{MW}$ Tjaereborg wind turbine and the 10MW DTU reference turbine, resepctively. The comparisons showed excellent agreement between the loadings using the new analytical model and the airfoil data based method for the Tjaereborg wind turbine. Analysing the analytical models, comparisons were also carried out for both a stand-alone wind turbine and two rotors located in a row subject to the influence of the atmospheric boundary layer and turbulence intensity. For all cases, an excellent agreement was obtained between results obtained using the analytical form of the rotor and results 
obtained using the actual geometry and airfoil data. The comparison comprised rotor loanding as well as velocity profiles in the wake behind the rotors.

For the 10MW DTU reference rotor, the comparisons of the loading showed a very good agreement at the outer $60 \%$ of the rotor radius. In the inner $40 \%$ of the rotor, however, the comparison was less convincing. This was to be anticipated, as the rotor design of the DTU reference rotor deviates significantly from those traditionally used to design aerodynamically optimum rotors. For example, the geometry of the reference rotor was found to result in a local maximum load value at $r / R=0.33$, which cannot be captured by a constant circulation model corrected for root and tip effects. In spite of this, the resulting axial velocity distributions were seen to exhibit excellent agreement between the distributions based on computations using the reference turbine with airfoil data and those computed using the generic form of the surface forces.

Although the model is derived for rotors operating below the rated wind speed, i.e. for rotors operating at optimal conditions, it can in principle be applied to all wind speeds. However, to represent the full wind regime adequately, the model needs to be modified with a non-uniform circulation distribution. This will be the topic for a future development of the model.

\section{References}

[1] W.J.M. Rankine, On the mechanical principles of the action of propellors, Trans. Inst. Nav. Arch. 6, 13 (1865).

[2] R.E. Froude, On the part played in propulsion by differences in fluid pressure, Nav. Arch. 30, 390 (1889).

[3] A. Betz, Das Maximum der theoretisch möglichen Ausnützung des Windes durch Windmotoren, Zeitschrift für das gesamte Turbinenwesen, Heft 26 (1920).

[4] J.H. Horlock, Actuator disk theory, McGraw-Hill, Great Britain, 1978.

[5] H. Glauert, Aerodynamic Theory, edited by Durand, Volume IV division L-III, in The General Momentum Theory, Springer, Reprinted in 1963 as a Dover edition.

[6] J.N. Sørensen, General momentum theory for horizontal axis wind turbines. Research Topics in Wind Energy, Springer, 2016.

[7] J.N. Sørensen, A, Myken, A. Unsteady actuator disc model for horizontal axis wind turbines. J. Wind Eng. Ind. Aerodyn. 39 (1992) 139-149.

[8] J.N. Sørensen, C. Kock, A model for unsteady rotor aerodynamics. J. Wind Eng. Ind. Aerodyn. 58 (1995) 259-275.

[9] C. Masson, A. Smaïli, C. Leclerc, Aerodynamic Analysis of HAWT's Operating in Unsteady Conditions, Wind Energy, vol. 4, (2001), pp. 1-22.

[10] I. Ammara, C. Leclerc, C. Masson, C. A Viscous Three-Dimensional Differential/Actuator-Disk

Method for the Aerodynamic Analysis of Wind Farms. Journal of Solar Energy Engineering. Vol. 124, Issue 4(2002) 345-356.

[11] A. Jimenez, A. Crespo, E. Migoya, J. Garcia, Large-eddy simulation of spectral coherence in a wind turbine wake, Environmental Research Letters 3 (2008) 1-9.

[12] M. Calaf, C. Meneveau, J. Meyers, Large eddy simulation study of fully developed wind-turbine array boundary layers, Physics of Fluids 22 (2010).

[13] F. Porté-Agel, Y.T. Wu, H. Lu, R. Conzemius, Large-eddy simulation of atmospheric boundary layer flow through wind turbines and wind farms, J. Wind Eng. Ind. Aerodyn. 99 (2011) 154-168.

[14] J.N. Sørensen, W.Z. Shen, Numerical Modeling of Wind Turbine Wakes, J. Fluids Eng.124(2) (2002) 393-399. doi:10.1115/1.1471361. 
[15] W.Z. Shen, J.H. Zhang, J.N. Sørensen, The Actuator Surface Model: A New Navier-Stokes Based Model for Rotor Computations', J. Sol. Energy Eng. 131(1) (2009) 011002. DOI:

http://dx.doi.org/10.1115/1.3027502.

[16] N. Simisiroglou, S. Sarmast, S.-P. Breton, S. Ivanell, 2016. Validation of the actuator disc approach in PHOENICS using small scale model wind turbines. J. Physics: Conference Series 753, 032028. IOP Publishing.Doi:10.1088/1742-6596/753/3/032028.

[17] P. van der Laan, N.N. Sørensen, P.-E. Réthoré, J. Mann, M.C. Kelly, N.Troldborg, The $k-\varepsilon-f_{p}$ model applied to double wind turbine wakes using different actuator disk force methods, Wind Energy vol. 18(12) (2015) 2065-2084.

[18] J.A. Michelsen, Basis3D - A Platform for Development of Multiblock PDE Solvers, Tech. Rep. AFM 92-05. Technical University of Denmark, 1992.

[19] J.A. Michelsen, Block Structured Multigrid Solution of 2D and 3D Elliptic PDE's, Tech. Rep. AFM 9406. Technical University of Denmark, 1994.

[20] N.N. Sørensen, General purpose flow solver applied to flow over hills, PhD thesis, Risø National Laboratory, 1995.

[21] N. Troldborg, J.N. Sørensen, R. Mikkelsen, R., N.N. Sørensen, A simple atmospheric boundary layer model applied to large eddy simulations of wind turbine wakes, Wind Energy 17 (2014) 657-669.

[22] J. Mann, The spatial structure of neutral atmospheric surface-layer turbulence, J. Fluid Mech. 273 (1994) 141-168.

[23] J. Mann, J. Wind field simulation, Probabilistic Engineering Mechanics 13 (1998) 269-282.

[24] R. Mikkelsen, Actuator Disc Methods Applied To Wind Turbines, MEK-FM-PHD 2003-02. Technical University of Denmark, 2003.

[25] A. Betz, Schraubenpropeller mit geringstem Energieverlust. Mit einem Zusatz von 1. Prandtl.

Nachrichten von der Gesellschaft der Wissenschaften zu Göttingen, Mathematisch-Physikalische Klasse 1919: 193-217, 1919.

[26] J.M. Delery, Aspects of vortex breakdown, Prog. Aerospace Sci. Vol. 30 (1994) 1-59.

[27] P. Friis, The Tjæreborg wind turbine. Final Report, Elsamproject A/S, Fredericia, Denmark. Report NEI-DK-1018. 1992 (downloaded from National Technical Reports Library (NTRL):

https://ntrl.ntis.gov/NTRL/dashboard/searchResults/titleDetail/DE93752793.xhtml).

[28] C. Bak, F. Zahle, R. Bitsche, T. Kim, A. Yde, L.C. Henriksen, M.H. Hansen, J.P.A.A. Blasques, M. Gaunaa, A. Natarajan, The DTU 10-MW Reference Wind Turbine, Technical university of Denmark, 2013. 